\title{
Towards practical classical processing for the surface code: timing analysis
}

\author{
Austin G. Fowler, Adam C. Whiteside, Lloyd C. L. Hollenberg \\ Centre for Quantum Computation and Communication Technology, \\ School of Physics, The University of Melbourne, Victoria 3010, Australia
}

(Dated: May 21, 2018)

\begin{abstract}
Topological quantum error correction codes have high thresholds and are well suited to physical implementation. The minimum weight perfect matching algorithm can be used to efficiently handle errors in such codes. We perform a timing analysis of our current implementation of the minimum weight perfect matching algorithm. Our implementation performs the classical processing associated with an $n \times n$ lattice of qubits realizing a square surface code storing a single logical qubit of information in a fault-tolerant manner. We empirically demonstrate that our implementation requires only $O\left(n^{2}\right)$ average time per round of error correction for code distances ranging from 4 to 512 and a range of depolarizing error rates. We also describe tests we have performed to verify that it always obtains a true minimum weight perfect matching.
\end{abstract}

\section{INTRODUCTION}

Quantum computers promise efficient factoring [1], efficient simulation of quantum systems [2, and the efficient solution of many other classically intractable problems [3]. The primary barrier to the realization of a quantum computer is the physical realization of quantum gates with sufficiently low error to enable quantum error correction to be used. Topological quantum error correction (TQEC) codes can tolerate error rates of order $1 \%$ [4, 5] and require only 2-D nearest neighbor interactions, both physically reasonable targets, however the classical processing associated with the error correction is highly nontrivial. Without significant future effort, the classical processing will almost certainly limit the speed of any quantum computer, particularly one with intrinsically fast quantum gates.

In this work, we present a timing analysis of our software performing the classical processing associated with TQEC. This software is by orders of magnitude the fastest currently available. We will review the necessary aspects of the surface code [6, 7, fault-tolerant schemes built on the surface code 8 , 10, and our classical processing algorithm [5] as required. Our goal is to analyze in detail the performance and correctness of our implementation of this algorithm. This implementation is contained in a library match.c and called by our tool Autotune [11, which is designed to prepare a graph problem tailored to arbitrary hardware running a surface code family TQEC scheme.

The discussion is organized as follows. In Section II the basic structure and functionality of our software is described. The library match.c, which performs minimum weight perfect matching [12, 13] is described in more detail in Section III Two versions are discussed. An example of the faster version of the algorithm in action is provided in Section IV] The probability of logical errors in the surface code as a function of the physical error rate $p$ is discussed in Section V. Formatted timing data is presented in Section VI. Complete raw timing data can be found in the Supplementary Material. Section VII summarizes and points to further work.
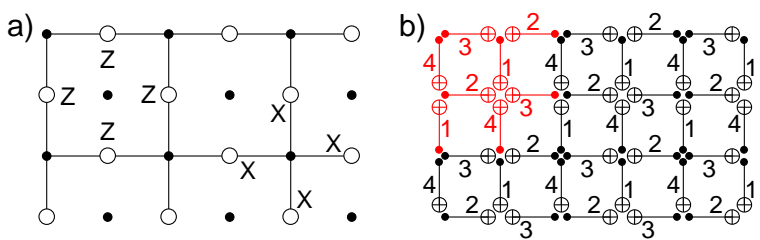

FIG. 1: (Color online) a) 2-D lattice of data qubits (circles) and syndrome qubits (dots) and examples of the data qubit stabilizers. b) Sequence of CNOTs permitting simultaneous measurement of all stabilizers. Numbers indicate the relative timing of gates. The highlighted gates can be tiled to fill the plane.

\section{OVERVIEW}

Our simulation suite of software is designed to handle arbitrary hardware with arbitrary stochastic error models, however we shall focus on a simple 2-D square lattice of qubits and a standard depolarizing channel for each quantum gate for the purposes of benchmarking and demonstrating correctness. Specifically, we shall study the case of no initialization surface code error detection 44. A small section of the 2-D array of data and syndrome qubits of the surface code and the required cyclic sequence of CNOTs to simultaneously measure all stabilizers [14] is shown in Fig. 1. At the end of each cycle, all syndrome qubits are measured in the $X$ or $Z$ basis according to whether they are being used to measure $X$ or $Z$ stabilizers, respectively.

Random Pauli errors are generated and propagated using a Pauli frame. When errors lead to syndrome measurement value changes, graph vertices are generated at these space-time locations. By pre-analyzing all possible single error processes [4, 11, an underlying lattice of dots and lines is also prepared with dots at every location a vertex could potentially be generated and lines between every pair of locations that could have vertices generated 


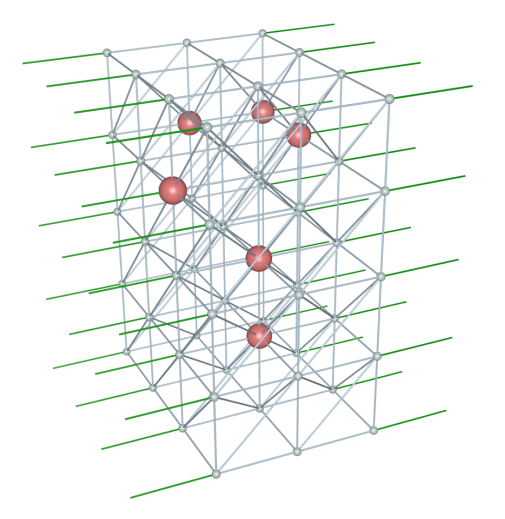

FIG. 2: (Color online) Distance 4 example of a lattice of dots and lines with stochastically generated vertices. The distance of a surface code if the length in lines of the shortest topologically nontrivial path, in this case any path connecting opposing boundaries. Dots (small balls) correspond to space-time locations where the endpoints of error chains could potentially be detected. Vertices (large balls) correspond to space-time locations where error chain end points have been detected. Light cylinders link pairs of dots where a pair of vertices could be generated by a single error. Dark cylinders link spatial boundaries to a single dot where a single vertex could be generated by a single error.

by a single error. The first order probability $p_{\text {line }}$ of each line is calculated and a weight $w=-\ln \left(p_{\text {line }}\right)$ stored in each line. This is done so that a large positive weight is associated with any line of low probability, ensuring that an algorithm matching vertices impairs using paths of lines with minimum total weight will tend to avoid using low probability lines. Furthermore, a multiple line path will have a weight related to the product of probabilities of its constituent lines. A lattice of dots and lines and stochastically generated vertices (from surface code simulation) is shown in Fig. 2 .

In many ways, a lattice plus vertices can be considered an implicit complete graph with an edge between any pair of vertices having weight equal to the minimum weight path between those vertices. The task is to match all vertices in pairs or to neighboring boundaries such that the total weight of all match paths is minimal. The basic algorithm that efficiently solves this problem given a standard graph is the minimum weight perfect matching algorithm [12, 13. We have extended this algorithm to include the concept of boundaries and permit new vertices to be dynamically added to the graph.

\section{MATCHING}

We have two operational versions of extended minimum weight perfect matching — complete match 4] which firstly constructs explicit edges between all pairs of vertices no more than approximately $d$ rounds of error

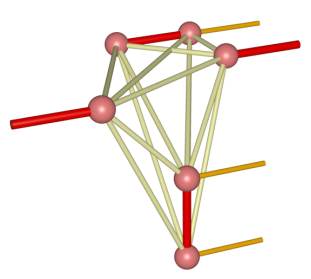

FIG. 3: (Color) Output of cmatch when given Fig. 2 as input. The underlying lattice is used to construct a complete graph and then discarded. Edges in the matching are shown in red.

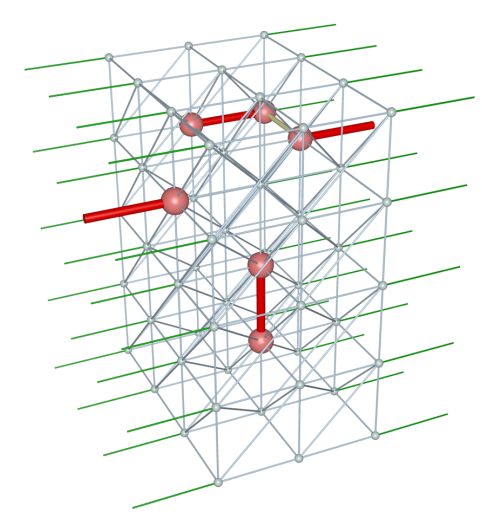

FIG. 4: (Color) Output of eodmatch when given Fig. 2 as input. Note that only one edge other than those ultimately included in the matching has been created (just visible between the top right two vertices).

correction apart, and edges on demand match [5] which only constructs a small number of local edges and adds further edges to the problem as required. The graphs and matchings generated by complete match (cmatch) and edges on demand match (eodmatch) given Fig. 2 as input are shown in Fig. 3 and Fig. 4 respectively. The total weight of matched edges in both cases is identical and in this case the matchings themselves are identical. We have tested cmatch and eodmatch on millions of varied problems, large and small, and always observed identical total weights, strongly implying both implementations are correct.

Cmatch obtains the true minimum weight perfect matching despite only including edges between vertices separated by a finite number of rounds. Vertices separated by a very large number of rounds are always cheaper to match to their nearest boundaries than to one another. By using the weights of the lines in the lattice, we calculate the minimum span of rounds to connect with edges to guarantee a minimum weight matching. Eodmatch also obtains a true minimum weight perfect matching as any required edge will eventually be included during execution. 


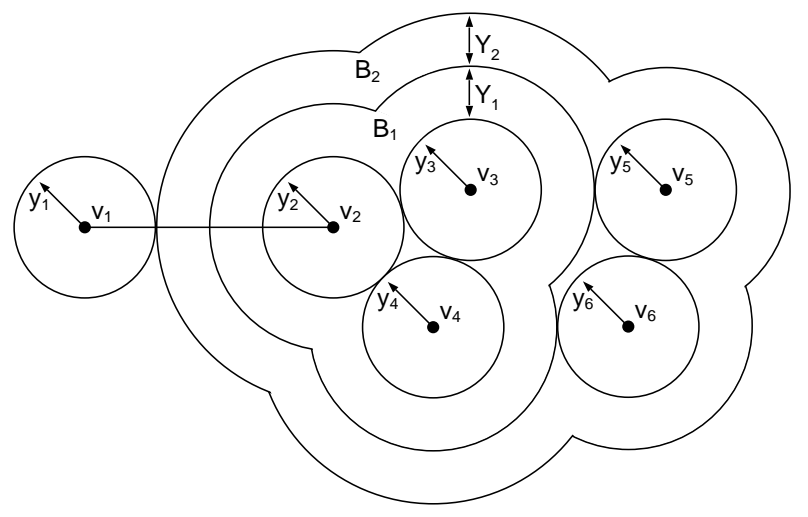

FIG. 5: An example of a tight edge. Edge $e_{12}$ has the property that $w_{12}-y_{1}-y_{2}-Y_{1}-Y_{2}=0$.

We now describe the eodmatch algorithm. Some definitions are required. Let $G$ be a graph with vertices $\left\{v_{i}\right\}$, edges $\left\{e_{i j}\right\}$, and edge weights $\left\{w_{i j}\right\}$. The graphs we use in eodmatch are implicitly complete, with the weight of an edge between any given pair of vertices defined to be the weight of a minimum weight path between those vertices, and the weight of any edge connecting a vertex to a nearby boundary defined similarly. As such, we shall describe the algorithm as though we have a complete graph. The process of dynamically adding the required edges is just a technical detail.

Associate with each vertex $v_{i}$ a variable $y_{i}$, which can be thought of as the radius of a ball centered at $v_{i}$. Odd sets of vertices can also be made into blossoms $B_{k}$ that have their own variables $Y_{k}$, which can be thought of as the width of shell around every object in $B_{k}$. If a pair of blossoms intersect, then one is contained in the other. Define an edge $e_{i j}$ to be tight if $w_{i j}-y_{i}-y_{j}-\sum Y_{k}=0$, where the sum is over $k$ such that exclusively $v_{i}$ or $v_{j}$ is in $B_{k}$. This condition is pictorially depicted in Fig. 5 .

Define a node to be a vertex or blossom. Allow edges to possess a label matched or unmatched. Define a blossom to be unmatched if it contains a vertex not incident on a matched edge. An alternating tree is a tree of nodes rooted on an unmatched node such that every path of edges from the root node to a leaf node consists of alternating unmatched and matched edges. Alternating trees can only branch from the root and every second node from the root. Define branching nodes to be outer. Define all other nodes in the alternating tree to be inner. Fig. 6 shows all necessary alternating tree manipulations.

Given a weighted graph $G$, the following algorithm finds a minimum weight perfect matching.

1. If there are no unmatched vertices, return the list of matched edges.

2. Choose an unmatched vertex $v$ to be the root of an alternating tree.
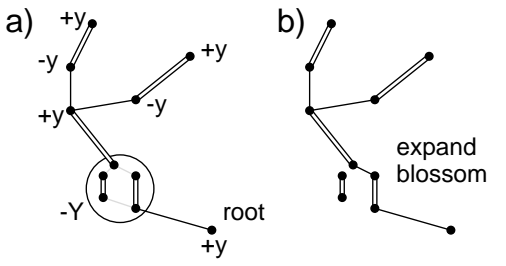

d)

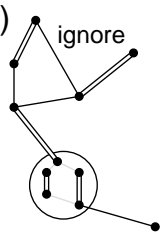

e)

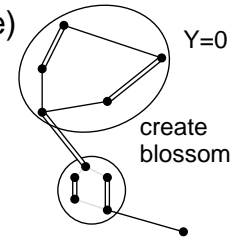

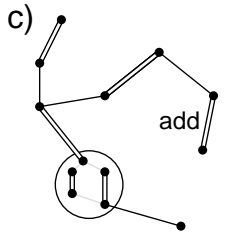

f)

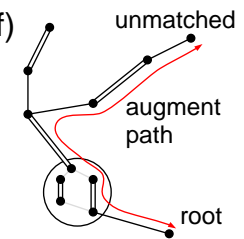

FIG. 6: (Color online) All required alternating tree manipulations. a) Increase outer node and decrease inner node $y$ values (or $Y$ if the node is a blossom), which will maintain the tightness of all tree edges and potentially creating new tight edges connected to at least one outer node. b) Inner blossoms with $Y=0$ can be expanded into multiple inner and outer nodes and potentially some nodes that are no longer part of the tree. c) Outer-matched tight edges can be used to grow the alternating tree. d) Outer-inner tight edges can be ignored as they never grow tighter. e) Outer-outer tight edges make cycles that can be used to form blossoms. f) When another unmatched vertex $v$ is found, or an edge to a boundary $b$, the path from the unmatched vertex within the root node through the alternating tree to $v$ or $b$ is augmented, meaning matched edges become unmatched and unmatched edges become matched. This strictly increases the total number of matched vertices.

3. If no edges emanating from the outer nodes of the alternating tree are tight, henceforth called $O$-tight edges, increase the value of $y$ or $Y$ associated with each outer node while simultaneously decreasing the value of $y$ or $Y$ associated with each inner node until an edge becomes $O$-tight, or an inner blossom node $Y$ variable becomes 0 (Fig. 6a).

4. If an inner blossom node $Y$ variable becomes 0 and there are still no $O$-tight edges, expand that blossom and return to 3 (Fig. 6b).

5. Choose an $O$-tight edge $e$.

6. If $e$ leads to a matched node not already in the alternating tree, add the relevant unmatched and matched edge and associated nodes to the alternating tree and return to 3 (Fig. 6r).

7. If $e$ leads to an inner node, mark $e$ so it is not considered again during the growth of this alternating tree and return to 3 (Fig. 6 d).

8. If $e$ leads to an outer node, add the unmatched edge to the alternating tree. There will now be a cycle of odd length. Collapse this cycle into a 
new blossom and associate a new variable $Y=0$ (Fig. 6e). Return to 3 .

9. If $e$ leads to an unmatched vertex or boundary, add $e$ to the alternating tree and augment the path (unmatched $\leftrightarrow$ matched) from the unmatched vertex within the root node to the end of $e$ (Fig. 6f). Destroy the alternating tree, keeping any newly formed blossoms. Return to 1 .

On average, the algorithm only needs to consider a small local region around each vertex to find another unmatched vertex to pair with. This is a property of the graphs associated with topological QEC only, as the probability of needing to consider an edge of length $l$ decreases exponentially with $l$. This ensures that the runtime is $O\left(n^{2}\right)$, and that the algorithm can be parallelized to achieve $O(1)$ processing per round.

\section{EODMATCH EXAMPLE}

The rules of the previous section are far from intuitive. Let's consider a simple 1-D chain of qubits suffering errors and generating vertices in space and time. Let's assume the underlying lattice is square. Fig. 7 a shows a possible current state of the matching algorithm, with matched vertices far in the past and unmatched vertices in the present and recent past. The goal is to match as many vertices as possible in the active region (between the horizontal dashed lines) without using any data that is too new. The window that defines the active region rolls forward as additional vertices are generated by the quantum computer. The first vertex chosen for matching is indicated with an arrow. It does not matter which vertex is chosen in the active region, however our algorithm has a preference for vertices further in the past.

Fig. $7 \mathrm{p}$ shows a shaded exploratory region around the chosen vertex. This is constructed by performing a breadth first search through the lattice local to the vertex. When any other object is encountered, whether it be a boundary, another exploratory region or another vertex, expansion is halted. In this case, two unmatched vertices and one exploratory region simultaneously terminate expansion. One of these vertices is chosen to be matched to as shown in Fig. 7k. It does not matter which vertex is chosen, both are valid choices that would lead to a minimum weight perfect matching being obtained. The next vertex is chosen.

In Fig. 7d, when exploration around the chosen vertex terminates, a matched vertex is encountered and no unmatched vertices. This necessitates the construction of an alternating tree. An alternating tree is a tree with alternating unmatched and matched edges. Alternating trees are only allowed to branch at the root and every second node from the root. Branching nodes are called outer nodes, non-branching nodes are called inner nodes. The alternating tree constructed in Fig. $7 \mathrm{~d}$ consists of three nodes, all of which are simple vertices. We will encounter more complex alternating trees shortly.

Our algorithm attempts to expand the exploratory regions around each outer node and contract the exploratory regions around each inner node. This is impossible in this case as the two outer nodes are touching. Instead, a cycle is formed as shown in Fig. 8a. This cycle is collapsed to form a blossom, leaving an alternating tree with a single outer node that is a blossom containing three vertices.

The exploratory region around the sole outer node in the alternating tree is expanded until other objects are encountered (Fig. 8b). An unmatched vertex and a boundary are encountered. Two options are available. We could match the edge from the original root vertex to the vertex below it, unmatch the existing matched edge, and then match the resultant unmatched vertex to the nearby boundary. Alternatively, we can match the original root vertex to the newly encountered unmatched vertex. Since this is simpler, we choose this option, the execution of which is shown in Fig. 8s. The next unmatched vertex chosen is indicated by an arrow. In this case, no expansion of the exploratory region around the vertex is possible. One must instead immediately form an alternating tree consisting of three vertices (Fig. 8d).

The outer node exploratory regions are expanded while the inner node exploratory region is contracted (Fig. 99). This results in the outer node exploratory regions touching, forming a cycle and thus a blossom (Fig. 9p). This collapses the alternating tree to a single outer node consisting of a blossom containing three vertices.

The exploratory region around the single blossom outer node cannot be expanded, necessitating the creation of another alternating tree a blossom outer node, then a blossom inner node, then a vertex outer node (Fig. 9. The two outer exploratory regions can be expanded while the blossom inner node exploratory region is contracted, however this leads to exploration outside the active region (Fig. 9d). When this happens, we run our algorithm backwards to the beginning of the current matching attempt, which in this case is Fig. 8.

This example hopefully gives a flavor of the algorithm. The salient features we wish to convey to the reader are the algorithm's space-time locality and continuous processing nature. These features enable one to understand the parallelization of the algorithm. We shall explain this by analogy.

Imagine a box being filled with sand using a 2-D array of tubes. Each tube represents a processor. Imagine the rate of sand coming out of each tube represents the difficulty of the matching problem locally. A slower rate of flow implies higher local difficulty. The sand itself represents vertices that have been matched. The rate of flow of all tubes is set below the maximum possible - pauses are inserted in the algorithm such that it is possible for a tube to be run at greater than the standard rate should it be required. When the problem is locally hard, the rate of flow decreases and a hollow forms locally. When 
a.)

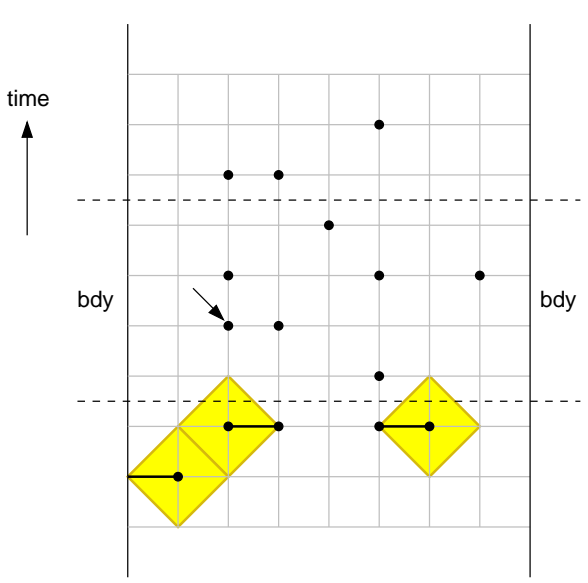

C.)

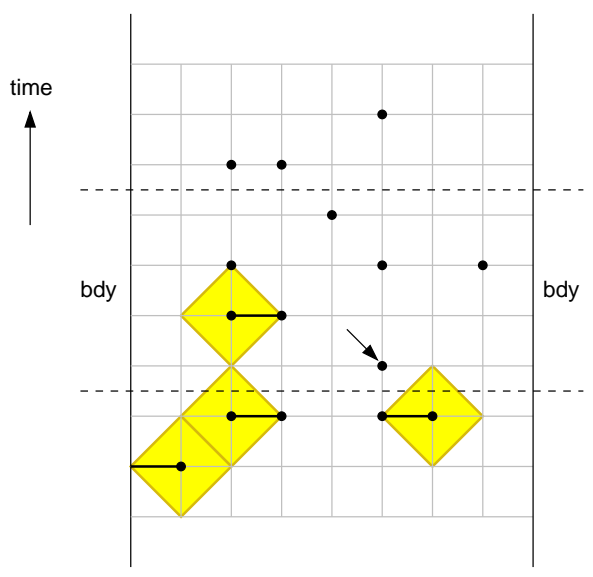

b.)

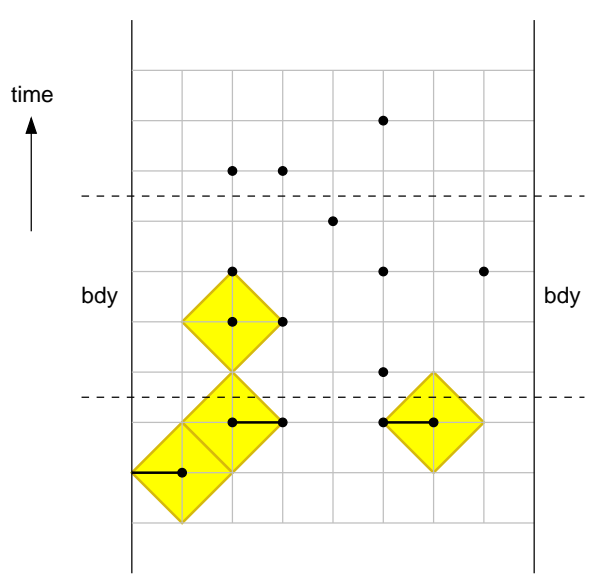

d.)

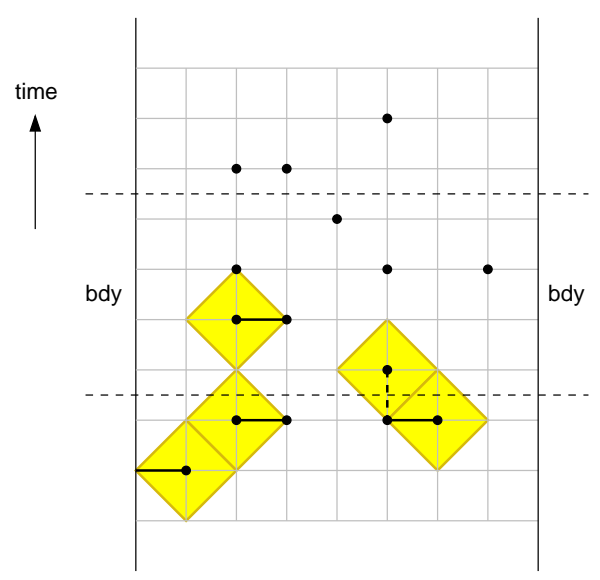

FIG. 7: (Color online) a.) Choose an unmatched vertex. b.) Expand exploratory region until other objects encountered. c.) Unmatched vertices encountered, choose one to match to. Choose another unmatched vertex. d.) Expand exploratory region until other objects encountered. Build alternating tree.

the difficulty of the problem returns to normal, which it must do on average, the rate of flow is increased above the standard rate to fill in this hollow. When the hollow is filled, the rate of flow is brought below the maximum possible again. Local difficulty does not result in global slowdown. Furthermore, surrounding tubes can assist in filling in the hollow. This simple picture explains how one can obtain a globally optimal solution of an infinite size problem in constant average time per round of processing, which is optimal.

Two other techniques for correcting errors in surface codes are being investigated, renormalization 15 and metropolis [16]. However, neither approach has been successfully applied to the realistic fault-tolerant case. Indeed, in the latest work of the authors of the renormalization approach, minimum weight perfect matching has been used to handle the fault-tolerant case [17. We are not hopeful that any technique other than matching can be comparably fast and effective in the fault-tolerant case.

\section{LOGICAL ERRORS}

Strong evidence of the correctness of eodmatch comes from studying the probability of logical error per round of error correction $\left(p_{L}\right)$ at depolarizing probabilities $p$ well below threshold. We calculate $p_{L}$ by simulating $t_{\text {check }}$ rounds of faulty quantum computer operation, then turning off errors, capping the matching problem with a perfect round of error correction, applying corrections, checking whether we have an odd or even number of errors along one of the boundaries and recording whether this is different to the previous time we checked. The perfect round of error correction is then undone and another $t_{\text {check }}$ faulty rounds simulated and the process repeated.

It may seem that the ideal value of $t_{\text {check }}$ is 1 to ensure that no logical errors are missed, however this is not 
a.)

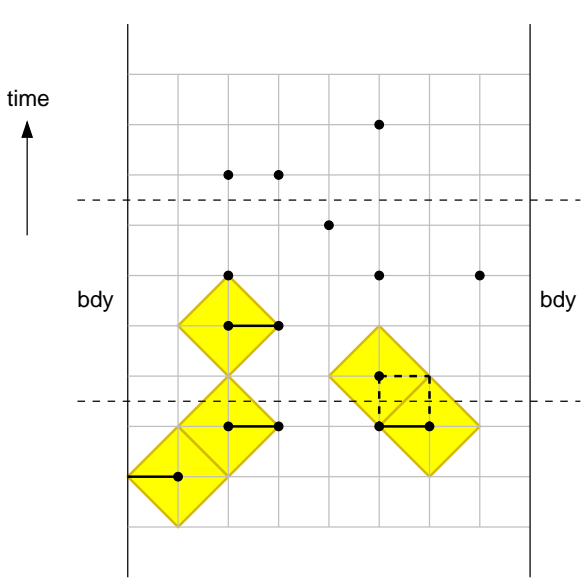

c.)

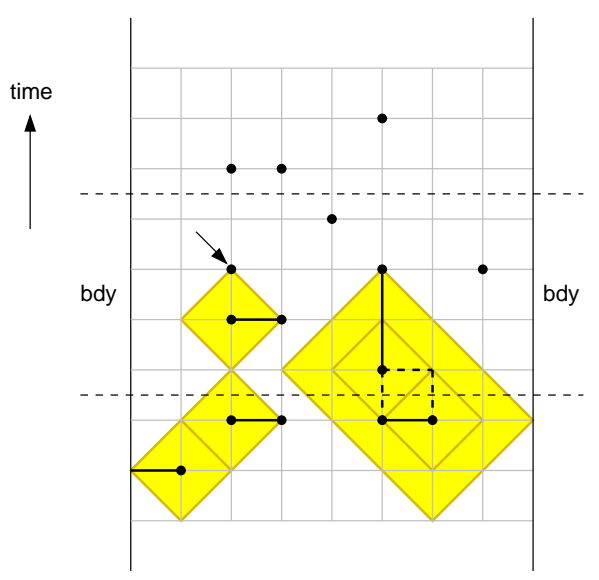

b.)

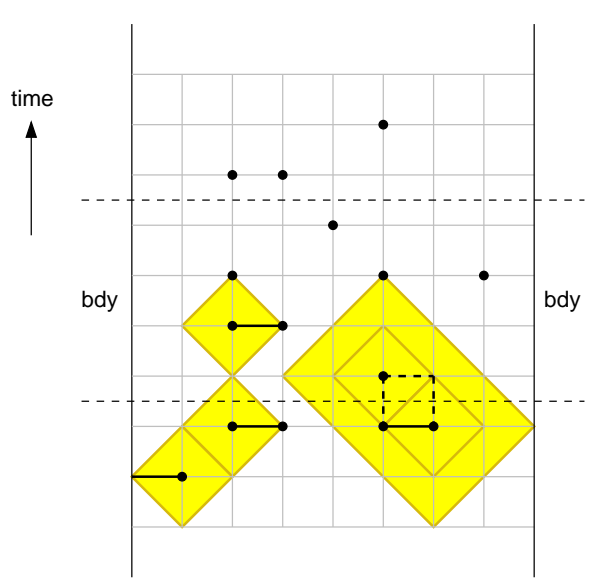

d.)

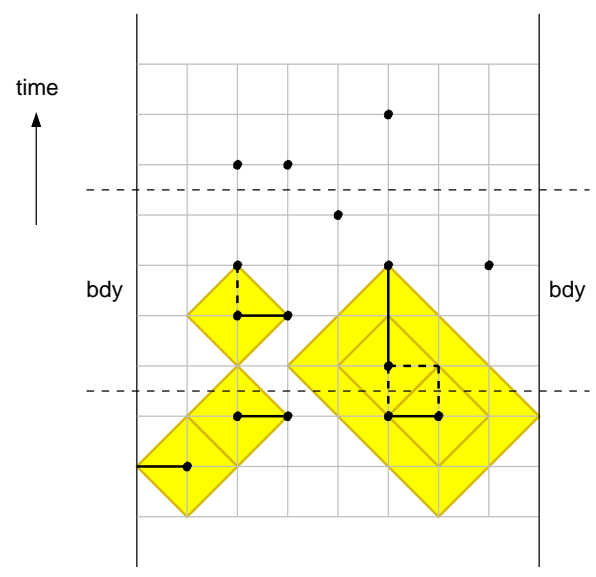

FIG. 8: (Color online) a.) Form blossom. b.) Expand exploratory region around blossom until other objects encountered. c.) Match to unmatched vertex. Choose another unmatched vertex. d.) Form alternating tree.

the case. We have observed that many combinations of errors lead to the observation of a logical error if a perfect round of error correction is inserted halfway through it, but no logical error if the perfect round of correction is sufficiently distant. With frequent checking this can mean a benign pattern of errors is counted as several logical errors. Instead, we typically use a value of $t_{\text {check }}$ such that a change in the parity of the number of errors observed along a boundary occurs approximately $10 \%$ of the time. We have empirically found that this leads to a logical error rate estimate robust to wide variations of $t_{\text {check }}$ about this value. The probability of a change per check is equal to the probability of an odd number of logical errors in $t_{\text {check }}$ rounds enabling $p_{L}$ to be easily calculated.

A distance $d$ code can reliably correct $\lfloor(d-1) / 2\rfloor$ errors. At low error rates $p$, clusters of errors are rare and well separated. The probability of suffering a logical error inducing cluster of $n_{d}=\lfloor(d+1) / 2\rfloor$ errors should therefore be $O\left(p^{n_{d}}\right)$ if the full distance of the code is be- ing realized. Figs. 10,11 show the complete set of data we have collected for the square surface code. Polynomials $A_{d} p^{n_{d}}$ are drawn through the lowest data point we were able to obtain for distances $3,5,7$ and 9 .

It is computationally expensive to obtain statistics at very low error rates and high distances as very few logical state changes are observed. It is also computationally expensive to obtain data at high error rates and high distances as the minimum weight perfect matching problem becomes more difficult around and above the threshold error rate $(0.9 \%$ [5]). The raw data used to generate Figs. 10 11, including timing information, can be found in the Supplementary Material.

The distance 3 and 5 dashed asymptotic curves in Figs. 1011 agree very well with the data. For higher distances, it is not currently possible to simulate a sufficiently large number of rounds of error correction to obtain sufficient information at low enough probabilities to achieve such tight agreement. Note that the high distance data curves approach the asymptotic curves with 
a.)

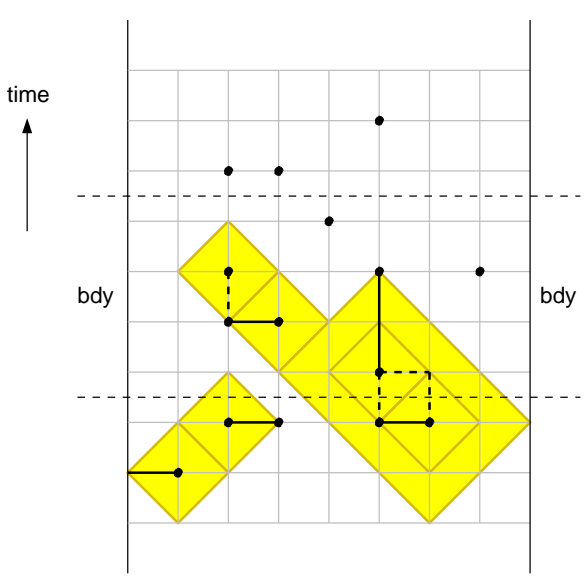

C.)

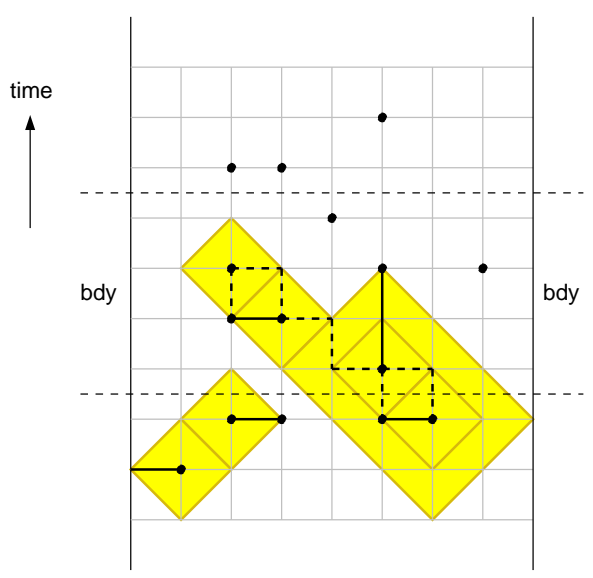

b.)

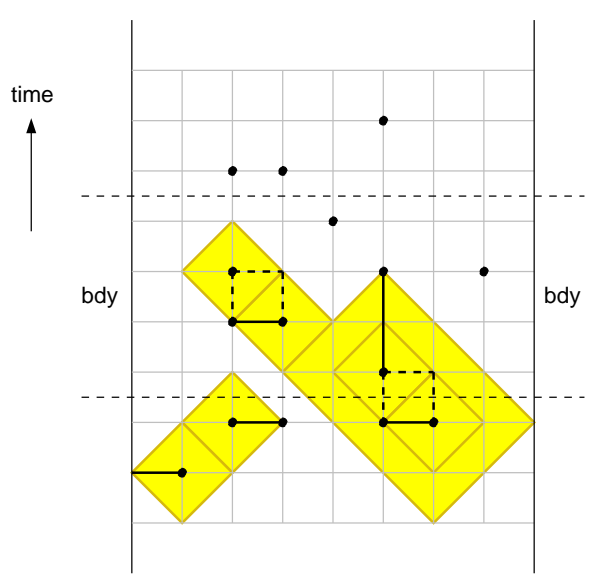

d.)

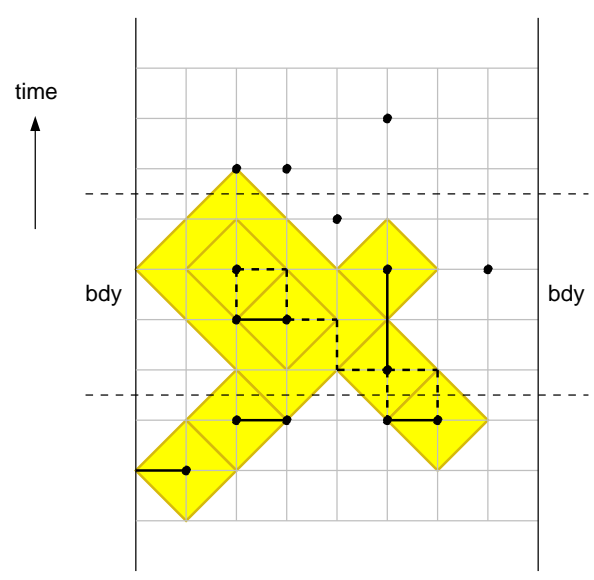

FIG. 9: (Color online) a.) Expand outer node exploratory regions, contract inner node exploratory region. b.) Form blossom. c.) Form alternating tree. d.) Expand outer node exploratory regions, contract inner node exploratory region. Forbidden region entered, reverse algorithm execution back to Fig. 8. Wait for additional data.

a steeper gradient, implying the surface code is capable of regularly correcting temporal clusters of errors containing more errors than the maximum guaranteed to be correctable. This is a generic feature of topological quantum error correction, as a large cluster of errors widely scattered across the code is not dangerous provided the cluster poorly resembles a topologically nontrivial chain of errors connecting distinct boundaries.

\section{TIMING}

The timing information in the Supplementary Material includes everything — initial bootup of the simulation, the simulation of the underlying quantum computer, problem generation, matching, perfect rounds of error correction to enable logical state change detection, and maintenance of an appropriate Pauli frame. Figs. 12 shows the amount of time devoted to each round of matching alone at three different error rates for distances $d=4,8,16, \ldots, 512$. The quadratic scaling of required time with distance is well demonstrated. At small $d$ nearby boundaries prevent the growth of large blossoms leading to increased performance. At very high $d$ memory access effects lead to a slight slowdown. Note that real computer systems are too complex to provide perfectly smooth graphs of time scaling even with long time averaging as the interplay of different levels of cache and RAM leads to measurable deviations from the ideal scaling.

To illustrate the complexity of modern computer memory systems, we have generated increasingly large arrays of random integers and calculated the time required to swap a constant large number $\left(10^{11}\right)$ of randomly chosen pairs of integers. The results are shown in Fig. 13. Ideally, a swap operation should be $O(1)$ independent of the array size. In practice, it can be seen that larger data sets lead to lower performance as CPU cache is exceeded. 


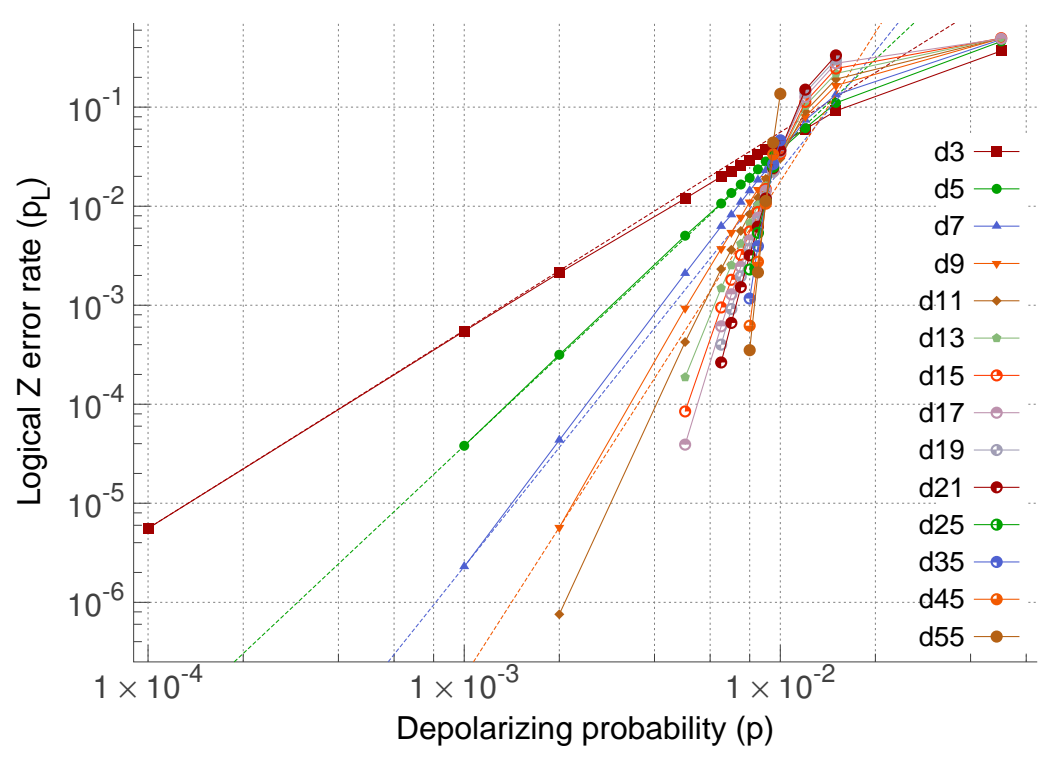

FIG. 10: (Color online) Logical $Z$ error rate per round of error correction for surface code distances $d$ and depolarizing noise probabilities $p$. Dashed lines indicate expected low $p$ asymptotic curves for $d=3,5,7$ and 9 .

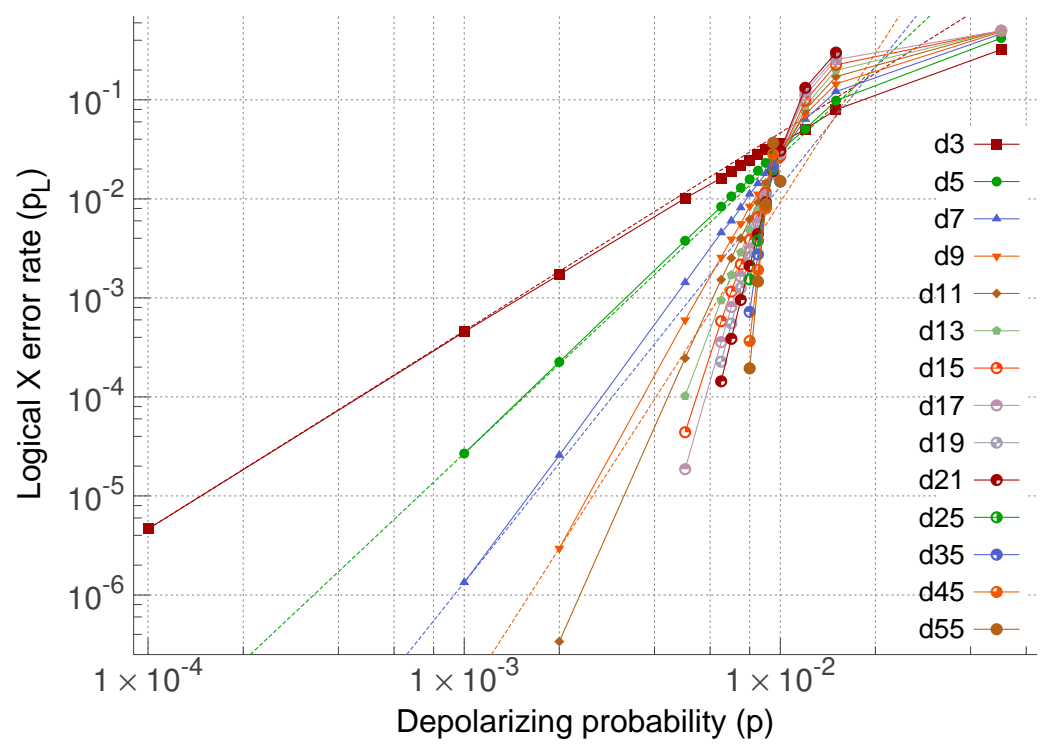

FIG. 11: (Color online) Logical $X$ error rate per round of error correction for surface code distances $d$ and depolarizing noise probabilities $p$. Dashed lines indicate expected low $p$ asymptotic curves for $d=3,5,7$ and 9 .

The data in Fig. 13 was generated by 16 core Intel Xeon $3.33 \mathrm{GHz}$ CPUs with $12 \mathrm{MB}$ of cache. Our matching code is more complex than this simple swap demonstration, with gradual delocalization of data as the data set increases in size. This leads to a gradual reduction of the probability of a single memory page load containing additional useful data.

\section{CONCLUSION}

After accounting for low distance nearby boundaries which limit the complexity of matching (making matching significantly faster) and high distance slower memory access (leading to a slight reduction in performance), Fig. 12 provide strong evidence supporting the claimed $O\left(d^{2}\right)$ runtime of our implementation of the algorithm described in [5]. A major future goal is to parallelize the algorithm and demonstrate an average processing time 


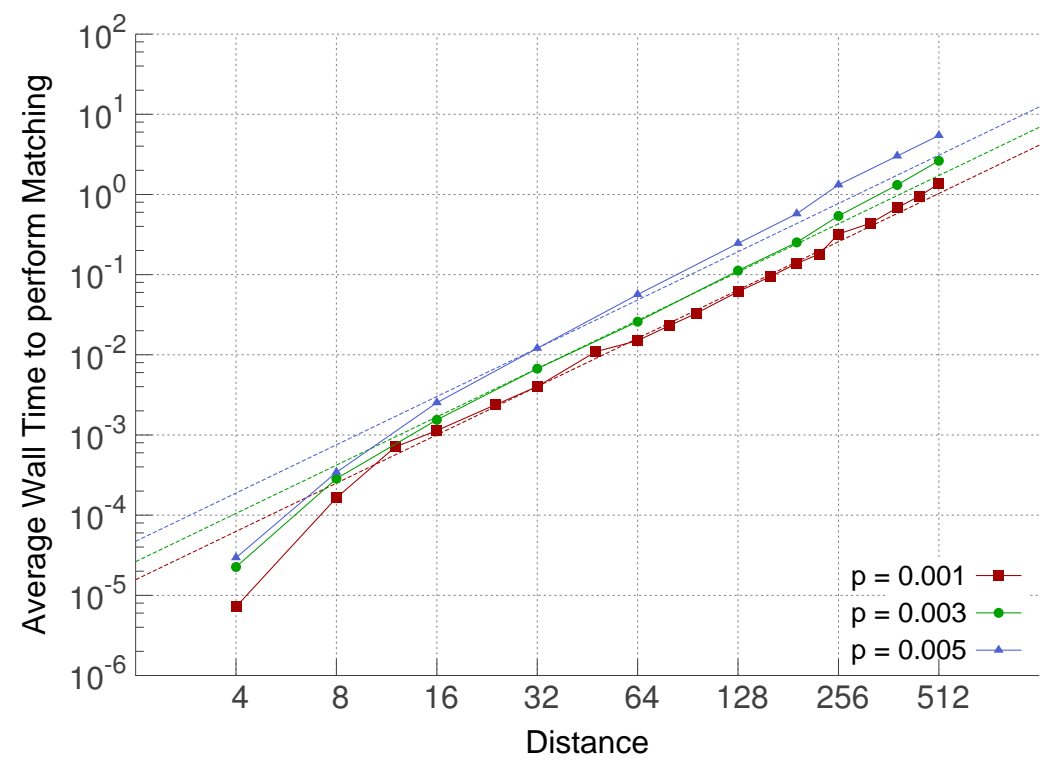

FIG. 12: (Color online) Amount time in seconds devoted to each round of matching when simulating a distance $d$ single logical qubit square surface code for depolarizing error rates $p$. Quadratic curves have been included for reference.

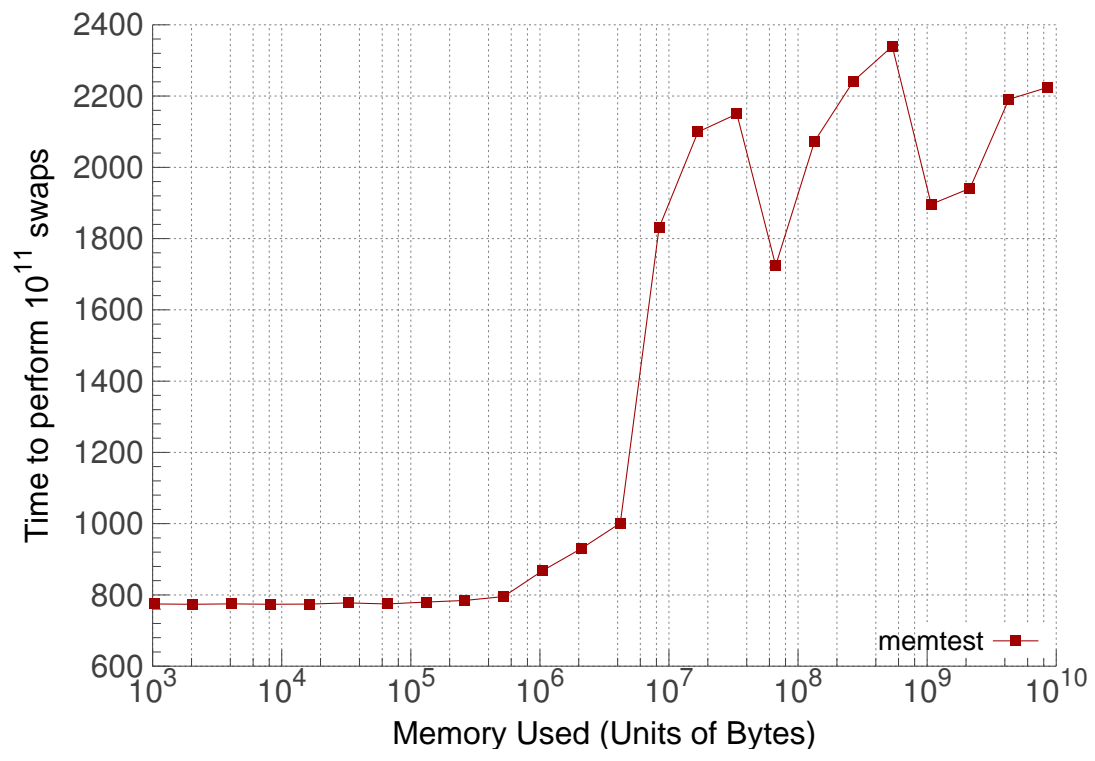

FIG. 13: (Color online) Average time in seconds required to perform $10^{11}$ swaps of randomly chosen pairs of integers in arrays of increasing size.

per round of error correction independent of the code size using constant computing resources per unit area.

\section{ACKNOWLEDGEMENTS}

We acknowledge support from the Australian Research Council Centre of Excellence for Quantum Computa- tion and Communication Technology (Project number CE110001027), and the US National Security Agency (NSA) and the Army Research Office (ARO) under contract number W911NF-08-1-0527. 


\section{SUPPLEMENTARY MATERIAL}

The raw data used to generate Figs. 10-11, including timing information, is listed below. The first number is the number of different distances (14). The second is the distance $d$ of the following block of data, the third is the number of different values $n$ of the depolarizing error rate $p$. The next $n$ lines list the value of $p, t_{\text {check }}$, the number of checks for $Z_{L}$ state changes, the number of checks for $X_{L}$ state changes, the observed number of $Z_{L}$ state changes, the observed number of $X_{L}$ state changes and finally the total number of CPU seconds devoted to the simulation of that $(d, p)$ pair. This basic structure is repeated for each distance. The last number in the file is the total number of CPU seconds devoted to creation of the entire file. Raw data was obtained using QuadCore AMD Opteron 2376 processors with $512 \mathrm{~Kb}$ of cache. Data has also been provided as simple text files.

14

3

15

0.0001257809399793997117541000037685 .82

0.00126194887948861181010000415 .42

0.0027093413934131201510000228 .69

0.005129169791696115401000033 .62

0.006579719897197120151000030 .32

0.0075113854113853115981000025 .31

0.00754121578121577116701000024 .84

0.0083142693142692116811000026 .08

0.00852184890184889118301000029 .62

0.0092164349164348116961000026 .93

0.00953107513107513114961000028 .59

0.0139895898957115231000021 .47

$0.0122103971 \quad 1039701175910000 \quad 22.25$

0.0151125634125633115361000027 .58

0.0513105131050114781000028 .47

5

14

0.0013861106980106979136281000047490 .43

0.00231415125115125113637100006568 .45

$\begin{array}{lllllll}0.005 & 26 & 111948 & 111947 & 12953 & 10000 & 653.26\end{array}$

0.006591422491422481252310000416 .50

$0.0076165703 \quad 165702 \quad 1262610000359.61$

$0.00756137318137317 \quad 12553 \quad 10000 \quad 308.21$

$0.008 \quad 6 \quad 114309 \quad 114308 \quad 12021 \quad 10000 \quad 325.45$

0.008541374681374671206110000329 .63

$0.0093 \quad 151197 \quad 151196 \quad 12121 \quad 10000 \quad 336.85$

0.009531313641313641191410000304 .38

0.0131162161162151189610000337 .23

0.01221036591036581195010000332 .92

0.01511018381018371126510000269 .22

0.05123807238061097510000544 .14

7

14

0.0016549312465212465216178100003521570 .27

$0.0023731 \quad 1146021146021583710001 \quad 165464.09$

0.00588897788977713864100006818 .24

$0.006513 \quad 178531 \quad 17853013492 \quad 10000 \quad 3272.27$

0.0071810258710258613184100001624 .85

0.00751013193513193413115100001651 .60

$0.0086 \quad 15782015781912635100001387.70$

$0.00855 \quad 148477 \quad 148476 \quad 12683 \quad 100001220.78$

$0.0094146237 \quad 14623612497 \quad 100001470.92$

0.0095412072412072412117100002172 .96

0.01312688912688812183100001086 .01

0.012115718215718111622100001510 .58
0.0151823468234511007100001145 .62 $\begin{array}{lllllll}0.05121522 & 121521 & 10388 & 10000 & 1717.54\end{array}$ 9

13

0.0023186211633311633317719100014289126 .50

$0.005134134774 \quad 134773 \quad 14912 \quad 10000 \quad 34427.08$

$0.006531 \quad 13537213537114001100009139.10$

$0.007 \quad 17 \quad 159583 \quad 159582 \quad 13532100006835.25$

$0.007513 \quad 14648314648213431 \quad 100005371.73$

0.008620635920635812936100004678 .65

$0.0085615738215738112947 \quad 10000 \quad 3371.82$

0.009612237112237012735100002893 .09

0.0095316320716320712108100002943 .78

0.01217791917791812051100005089 .92

0.012114023414023311265100004300 .04

0.0151689816898011432100003114 .11

0.051205482054710181100005762 .83

11

13

$0.002100002970715297071522288 \quad 1001460628087.09$

$0.005442102391 \quad 10239016041 \quad 10000 \quad 116498.16$

$\begin{array}{llllllll}0.0065 & 39 & 177762 & 177761 & 14738 & 10000 & 27844.55\end{array}$

$0.00734 \quad 126474126473138821000026712.36$

$0.007518 \quad 149239149238137491000015172.28$

$0.008 \quad 1017013917013813115100009234.01$

$0.00858144574 \quad 144573 \quad 12947 \quad 10000 \quad 10480.43$

0.009323767123767012947100009925 .01

0.0095317169517169512202100009361 .08

0.01410785810785711928100006497 .89

$0.0121126752126751 \quad 11515100007390.47$

0.0151581625816111174100004262 .45

0.051199841998399361000012520 .50

13

12

0.0059371145721145721698010000603790 .28

$0.0065 \quad 58192423192422152661000078381.30$

$0.007321932401932391450210000 \quad 50053.45$

0.007526143566143565140551000034005 .52

0.00812180949180948136131000022861 .61

0.008510139111139110130251000021325 .58

$0.0095172414 \quad 172413129201000020014.77$

0.00955121057121057124001000013410 .58

0.013130958130957120651000011380 .03

0.0121115078115077119311000010931 .53

0.0151502825028111036100006670 .03

0.0511996419963100091000028043 .39

15

12

$0.00524561028921028901749210001 \quad 2173892.01$

0.00651511241431241431547110000222593 .70

$0.007 \quad 52 \quad 17628217628215132 \quad 10000115430.02$

$0.0075 \quad 28174215174215144151000078240.36$

$0.008 \quad 17 \quad 161912 \quad 161912 \quad 14015 \quad 10000 \quad 52890.79$

0.008511148576148576131021000054511 .94

$0.0095 \quad 18142218142212771 \quad 1000034858.01$

$0.009541462411462411241610000 \quad 30968.27$

0.013129322129322122531000028182 .51

0.0121101864101864114981000017875 .60

$0.01514424744247 \quad 108631000011634.90$

0.051198641986499031000098384 .02

17

12

0.005588510161310161318732100006704496 .11

0.00652751119391119391602110000516700 .36

0.007891490291490291527710000256345 .82

$0.0075 \quad 541231381231381429110000 \quad 126590.64$

$0.00823148027 \quad 148027139611000082716.06$

$0.0085 \quad 15 \quad 12726512726513161 \quad 1000056207.63$

0.0095191569191569129611000045131 .54

0.00954148226148226123481000034119 .88 
0.012169382169382120101000046805 .81 0.01218993889938114081000024677 .61 0.01513925039250108921000017671 .96 0.0512001320013981810000132420 .21 19

10

0.00654201154091154091647410000963439 .12 $0.007 \quad 163 \quad 121377 \quad 121377 \quad 1565910000 \quad 533590.20$ 0.0075691257021257021487910000269356 .52 $0.008 \quad 469446794467 \quad 13698 \quad 10000144521.98$ 0.008520114779114779139361000082840 .47 $0.0098 \quad 142942 \quad 142942 \quad 12913 \quad 1000060109.55$ 0.00954146009146009123001000055487 .99 $0.013 \quad 1234221234221236910000 \quad 47540.48$ 0.01218107281072114111000035343 .85 0.01513670536705111591000028088 .66 21

10

0.0065859914699146316689100002147913 .54

$0.007 \quad 226 \quad 124800124798 \quad 16152100001191853.64$ $0.0075107 \quad 1081721081721504810000472320.02$ $0.00844 \quad 1182091182091448010000 \quad 276634.69$ 0.0085191288411288411365410000125697 .36 0.0098144541144541127161000093677 .72 0.009531822941822941263010000170367 .06 $0.013115647 \quad 115647 \quad 118211000063634.76$ $0.0121756897568911377 \quad 1000052612.18$ 0.01513335533355111091000040737 .04 25

5

$0.008551285641285641435210000 \quad 507860.33$ 0.0085171651921651921388810000231570 .51 0.00991433321433321321110000175110 .91 $0.009531731401731401235210001 \quad 163829.39$ 0.011999011 .04 35

5

$0.00888 \quad 167587 \quad 16758715592 \quad 100002801803.43$ $0.0085 \quad 1426911526911514131 \quad 10000 \quad 1292528.08$ 0.00971769421769421282210000550365 .22 $0.0095 \quad 4 \quad 125106 \quad 1251061231410001437366.43$ $0.0123434 \quad 3 \quad 161.08$

45

5

0.008230128455128422159691000011334092 .96 0.00852720319620319614039100002845252 .53 $0.0099147701 \quad 147701 \quad 12962100001587903.33$ 0.009531251961251961168410001944033 .23 0.0111313016 .30 55

5

0.008197285003284944184331048937296797 .55 $0.0085342107992107851434010000 \quad 7669758.24$ 0.009717007217007212541100003025099 .15 0.0095214084314084011836100002813159 .55 0.0116666912038 .71

161469013.53

For convenience, we also include below the processed raw data with checks and changes converted into probabilities of logical error. Second column is the probability of $Z_{L}$ error per round of error correction. Third column is the same data for $X_{L}$.

14

3

15

$1.000000 e-04 \quad 5.581956 e-06 \quad 4.639970 e-06$

$1.000000 \mathrm{e}-03 \quad 5.480776 \mathrm{e}-04 \quad 4.532594 \mathrm{e}-04$

$2.000000 \mathrm{e}-03 \quad 2.119693 \mathrm{e}-03 \quad 1.717972 \mathrm{e}-03$
$5.000000 e-03 \quad 1.193646 e-02 \quad 1.014745 e-02$ $6.500000 \mathrm{e}-03 \quad 1.987923 \mathrm{e}-02 \quad 1.618780 \mathrm{e}-02$ $7.000000 e-03 \quad 2.227094 e-02 \quad 1.894943 e-02$ $7.500000 \mathrm{e}-03 \quad 2.594789 \mathrm{e}-02 \quad 2.196915 \mathrm{e}-02$ $8.000000 \mathrm{e}-03 \quad 2.892852 \mathrm{e}-02 \quad 2.454551 \mathrm{e}-02$ $8.500000 \mathrm{e}-03 \quad 3.308666 \mathrm{e}-02 \quad 2.781694 \mathrm{e}-02$ $9.000000 \mathrm{e}-03 \quad 3.694796 \mathrm{e}-02 \quad 3.140969 \mathrm{e}-02$ $9.500000 \mathrm{e}-03 \quad 3.853583 \mathrm{e}-02 \quad 3.315384 \mathrm{e}-02$ $1.000000 \mathrm{e}-02 \quad 4.229061 \mathrm{e}-02 \quad 3.624911 \mathrm{e}-02$ $1.200000 \mathrm{e}-02 \quad 6.016994 \mathrm{e}-02 \quad 5.065678 \mathrm{e}-02$ $1.500000 \mathrm{e}-02 \quad 9.182239 \mathrm{e}-02 \quad 7.959676 \mathrm{e}-02$ $5.000000 e-02 \quad 3.696508 \mathrm{e}-01 \quad 3.220606 \mathrm{e}-01$ 5

14

$1.000000 \mathrm{e}-03 \quad 3.808086 \mathrm{e}-05 \quad 2.680139 \mathrm{e}-05$ $2.000000 \mathrm{e}-03 \quad 3.165315 \mathrm{e}-04 \quad 2.257910 \mathrm{e}-04$ $5.000000 \mathrm{e}-03 \quad 5.035972 \mathrm{e}-03 \quad 3.770583 \mathrm{e}-03$ $6.500000 \mathrm{e}-03 \quad 1.064455 \mathrm{e}-02 \quad 8.347346 \mathrm{e}-03$ $7.000000 \mathrm{e}-03 \quad 1.359007 \mathrm{e}-02 \quad 1.060487 \mathrm{e}-02$ $\begin{array}{lll}7.500000 \mathrm{e}-03 & 1.654580 \mathrm{e}-02 & 1.294715 \mathrm{e}-02\end{array}$ $8.000000 \mathrm{e}-03 \quad 1.929566 \mathrm{e}-02 \quad 1.577339 \mathrm{e}-02$ $8.500000 \mathrm{e}-032.354591 \mathrm{e}-02 \quad 1.927205 \mathrm{e}-02$ $9.000000 \mathrm{e}-03 \quad 2.829314 \mathrm{e}-02 \quad 2.309704 \mathrm{e}-02$ $9.500000 \mathrm{e}-03 \quad 3.226927 \mathrm{e}-02 \quad 2.678389 \mathrm{e}-02$ $1.000000 \mathrm{e}-02 \quad 3.675606 \mathrm{e}-02 \quad 3.050584 \mathrm{e}-02$ $1.200000 \mathrm{e}-02 \quad 6.141257 \mathrm{e}-02 \quad 5.081799 \mathrm{e}-02$ $1.500000 \mathrm{e}-02 \quad 1.106172 \mathrm{e}-01 \quad 9.819651 \mathrm{e}-02$ $5.000000 \mathrm{e}-02 \quad 4.610004 \mathrm{e}-01 \quad 4.200611 \mathrm{e}-01$ 7

14

$1.000000 \mathrm{e}-03 \quad 2.294325 \mathrm{e}-06 \quad 1.335142 \mathrm{e}-06$ $2.000000 e-03 \quad 4.335013 e-05 \quad 2.570402 e-05$ $5.000000 e-03 \quad 2.094460 e-03 \quad 1.429906 e-03$ $6.500000 \mathrm{e}-03 \quad 6.262974 \mathrm{e}-03 \quad 4.548905 \mathrm{e}-03$ $7.000000 \mathrm{e}-03 \quad 8.185048 \mathrm{e}-03 \quad 5.987798 \mathrm{e}-03$ $7.500000 \mathrm{e}-03 \quad 1.096092 \mathrm{e}-02 \quad 8.152393 \mathrm{e}-03$ $8.000000 \mathrm{e}-03 \quad 1.433186 \mathrm{e}-02 \quad 1.116570 \mathrm{e}-02$ $8.500000 \mathrm{e}-03 \quad 1.838769 \mathrm{e}-02 \quad 1.426086 \mathrm{e}-02$ $9.000000 \mathrm{e}-03 \quad 2.288849 \mathrm{e}-02 \quad 1.804973 \mathrm{e}-02$ $9.500000 e-03 \quad 2.723837 e-02 \quad 2.213544 e-02$ $1.000000 \mathrm{e}-02 \quad 3.430413 \mathrm{e}-02 \quad 2.778531 \mathrm{e}-02$ $1.200000 \mathrm{e}-02 \quad 7.393956 \mathrm{e}-02 \quad 6.362081 \mathrm{e}-02$ $1.500000 \mathrm{e}-02 \quad 1.336675 \mathrm{e}-01 \quad 1.214404 \mathrm{e}-01$ $5.000000 \mathrm{e}-02 \quad 4.826679 \mathrm{e}-01 \quad 4.646626 \mathrm{e}-01$ 9

13

$2.000000 e-035.701198 \mathrm{e}-06 \quad 2.960670 \mathrm{e}-06$ $5.000000 \mathrm{e}-03 \quad 9.323964 \mathrm{e}-04 \quad 5.990253 \mathrm{e}-04$ $6.500000 \mathrm{e}-03 \quad 3.723894 \mathrm{e}-03 \quad 2.571852 \mathrm{e}-03$ $7.000000 \mathrm{e}-03 \quad 5.436050 \mathrm{e}-03 \quad 3.922925 \mathrm{e}-03$ $7.500000 \mathrm{e}-03 \quad 7.731204 \mathrm{e}-03 \quad 5.614499 \mathrm{e}-03$ $8.000000 \mathrm{e}-03 \quad 1.103955 \mathrm{e}-02 \quad 8.423489 \mathrm{e}-03$ $8.500000 \mathrm{e}-03 \quad 1.475789 \mathrm{e}-02 \quad 1.119869 \mathrm{e}-02$ $9.000000 \mathrm{e}-03 \quad 1.907401 \mathrm{e}-02 \quad 1.465237 \mathrm{e}-02$ $9.500000 \mathrm{e}-03 \quad 2.606438 \mathrm{e}-02 \quad 2.132012 \mathrm{e}-02$ $1.000000 \mathrm{e}-02 \quad 3.509833 \mathrm{e}-02 \quad 2.894034 \mathrm{e}-02$ $1.200000 \mathrm{e}-02 \quad 8.033013 \mathrm{e}-02 \quad 7.130980 \mathrm{e}-02$ $1.500000 \mathrm{e}-02 \quad 1.657271 \mathrm{e}-01 \quad 1.449695 \mathrm{e}-01$ $5.000000 \mathrm{e}-02 \quad 4.954739 \mathrm{e}-01 \quad 4.866886 \mathrm{e}-01$ 11

13

$2.000000 e-03 \quad 7.559446 e-07 \quad 3.382315 e-07$ $5.000000 \mathrm{e}-03 \quad 4.250431 \mathrm{e}-04 \quad 2.457825 \mathrm{e}-04$ $6.500000 \mathrm{e}-03 \quad 2.319008 \mathrm{e}-03 \quad 1.527896 \mathrm{e}-03$ $7.000000 \mathrm{e}-03 \quad 3.631596 \mathrm{e}-03 \quad 2.525026 \mathrm{e}-03$ $7.500000 \mathrm{e}-03 \quad 5.625133 \mathrm{e}-03 \quad 3.980921 \mathrm{e}-03$ $8.000000 e-03 \quad 8.302013 e-03 \quad 6.213838 e-03$ $8.500000 e-03 \quad 1.218413 e-02 \quad 9.219708 \mathrm{e}-03$ $9.000000 \mathrm{e}-03 \quad 1.886069 \mathrm{e}-02 \quad 1.443793 \mathrm{e}-02$ $9.500000 \mathrm{e}-032.490968 \mathrm{e}-02 \quad 2.022095 \mathrm{e}-02$ 
$1.000000 e-023.029043 e-022.499077 e-02$ $1.200000 \mathrm{e}-02 \quad 9.084678 \mathrm{e}-02 \quad 7.889485 \mathrm{e}-02$ $1.500000 \mathrm{e}-02 \quad 1.921186 \mathrm{e}-01 \quad 1.719365 \mathrm{e}-01$ $5.000000 \mathrm{e}-02 \quad 4.971981 \mathrm{e}-01 \quad 4.999981 \mathrm{e}-01$ 13

12

$5.000000 \mathrm{e}-031.875611 \mathrm{e}-04 \quad 1.023601 \mathrm{e}-04$ $6.500000 e-03 \quad 1.487202 e-03 \quad 9.451868 e-04$ $7.000000 \mathrm{e}-032.534618 \mathrm{e}-03 \quad 1.704212 \mathrm{e}-03$ $7.500000 e-03 \quad 4.172993 e-032.876705 e-03$ $8.000000 \mathrm{e}-03 \quad 6.748340 \mathrm{e}-03 \quad 4.856594 \mathrm{e}-03$ $8.500000 \mathrm{e}-031.026050 \mathrm{e}-027.700932 \mathrm{e}-03$ $9.000000 \mathrm{e}-031.597595 \mathrm{e}-021.217906 \mathrm{e}-02$ $9.500000 \mathrm{e}-03 \quad 2.240651 \mathrm{e}-02 \quad 1.773543 \mathrm{e}-02$ $1.000000 \mathrm{e}-02$ 3.281617e-02 2.687198e-02 $1.200000 \mathrm{e}-02 \quad 1.036773 \mathrm{e}-018.689857 \mathrm{e}-02$ $1.500000 \mathrm{e}-022.194815 \mathrm{e}-01 \quad 1.988821 \mathrm{e}-01$ $5.000000 \mathrm{e}-02 \quad 4.999981 \mathrm{e}-01 \quad 4.999981 \mathrm{e}-01$ 15

12

$5.000000 e-03 \quad 8.458677 e-05 \quad 4.400683 e-05$ $6.500000 \mathrm{e}-03 \quad 9.483575 \mathrm{e}-04 \quad 5.813465 \mathrm{e}-04$ $7.000000 \mathrm{e}-03 \quad 1.807830 \mathrm{e}-03 \quad 1.156577 \mathrm{e}-03$ $7.500000 \mathrm{e}-033.220028 \mathrm{e}-032.172807 \mathrm{e}-03$ $8.000000 e-035.559875 e-03 \quad 3.862835 e-03$ $8.500000 \mathrm{e}-038.742246 \mathrm{e}-036.528671 \mathrm{e}-03$ $9.000000 \mathrm{e}-03 \quad 1.494599 \mathrm{e}-02 \quad 1.154505 \mathrm{e}-02$ $9.500000 \mathrm{e}-032.272859 \mathrm{e}-02 \quad 1.804911 \mathrm{e}-02$ $1.000000 e-02 \quad 3.381841 e-022.723163 e-02$ $1.200000 \mathrm{e}-02$ 1.128764e-01 9.816980e-02 $1.500000 \mathrm{e}-02 \quad 2.455072 \mathrm{e}-012.260046 \mathrm{e}-01$ $5.000000 \mathrm{e}-02 \quad 4.985409 \mathrm{e}-01 \quad 4.999981 \mathrm{e}-01$ 17

12

$5.000000 \mathrm{e}-03 \quad 3.907789 \mathrm{e}-05 \quad 1.862184 \mathrm{e}-05$ $6.500000 \mathrm{e}-03 \quad 6.127439 \mathrm{e}-04 \quad 3.577438 \mathrm{e}-04$ $7.000000 \mathrm{e}-03 \quad 1.287325 \mathrm{e}-03 \quad 8.089195 \mathrm{e}-04$ $7.500000 \mathrm{e}-032.439529 \mathrm{e}-03 \quad 1.638397 \mathrm{e}-03$ $8.000000 \mathrm{e}-03 \quad 4.523496 \mathrm{e}-03 \quad 3.145585 \mathrm{e}-03$ $8.500000 \mathrm{e}-03 \quad 7.664518 \mathrm{e}-03 \quad 5.666593 \mathrm{e}-03$ $9.000000 \mathrm{e}-03 \quad 1.432955 \mathrm{e}-02 \quad 1.090556 \mathrm{e}-02$ $9.500000 \mathrm{e}-032.227061 \mathrm{e}-02 \quad 1.779357 \mathrm{e}-02$ $1.000000 e-023.680715 e-023.044608 e-02$ $1.200000 \mathrm{e}-021.268430 \mathrm{e}-01 \quad 1.111875 \mathrm{e}-01$ $1.500000 \mathrm{e}-022.775030 \mathrm{e}-012.547770 \mathrm{e}-01$ $5.000000 \mathrm{e}-02 \quad 4.905796 \mathrm{e}-01 \quad 4.996738 \mathrm{e}-01$ 19

10

$6.500000 \mathrm{e}-03 \quad 4.000280 \mathrm{e}-04 \quad 2.265075 \mathrm{e}-04$ $7.000000 \mathrm{e}-039.146123 \mathrm{e}-045.520112 \mathrm{e}-04$
$7.500000 e-031.953781 e-031.254145 e-03$ $8.000000 e-03 \quad 3.708987 e-032.579145 e-03$ $8.500000 e-03 \quad 6.906109 e-03 \quad 4.763698 e-03$ $9.000000 e-031.230079 e-029.332162 e-03$ $9.500000 \mathrm{e}-032.253916 \mathrm{e}-02 \quad 1.807942 \mathrm{e}-02$ $1.000000 e-02 \quad 3.592503 e-02 \quad 2.861376 e-02$ $1.200000 \mathrm{e}-021.407514 \mathrm{e}-01 \quad 1.233468 \mathrm{e}-01$ $1.500000 e-02 \quad 3.040190 e-012.724428 e-01$ 21

10

$6.500000 \mathrm{e}-032.641856 \mathrm{e}-04 \quad 1.436088 \mathrm{e}-04$ $7.000000 \mathrm{e}-03 \quad 6.622730 \mathrm{e}-04 \quad 3.862715 \mathrm{e}-04$ $7.500000 \mathrm{e}-03 \quad 1.521229 \mathrm{e}-03 \quad 9.543867 \mathrm{e}-04$ $8.000000 \mathrm{e}-03 \quad 3.183285 \mathrm{e}-032.101901 \mathrm{e}-03$ $8.500000 \mathrm{e}-03 \quad 6.229161 \mathrm{e}-03 \quad 4.419605 \mathrm{e}-03$ $9.000000 \mathrm{e}-03 \quad 1.195018 \mathrm{e}-02 \quad 9.221913 \mathrm{e}-03$ $9.500000 \mathrm{e}-03 \quad 2.425194 \mathrm{e}-02 \quad 1.899817 \mathrm{e}-02$ $1.000000 \mathrm{e}-02 \quad 3.669981 \mathrm{e}-02 \quad 3.066557 \mathrm{e}-02$ $1.200000 \mathrm{e}-02 \quad 1.503129 \mathrm{e}-01 \quad 1.321197 \mathrm{e}-01$ $1.500000 e-023.330526 e-012.998056 e-01$ 25

5

$8.000000 e-03 \quad 2.291625 e-03 \quad 1.534796 e-03$ $8.500000 e-035.385383 e-03 \quad 3.781245 e-03$ $9.000000 \mathrm{e}-03 \quad 1.119275 \mathrm{e}-02 \quad 8.279782 \mathrm{e}-03$ $9.500000 e-032.501067 e-02 \quad 2.004721 e-02$ $1.000000 \mathrm{e}-020.000000 \mathrm{e}+001.111112 \mathrm{e}-01$ 35

5

$8.000000 e-03 \quad 1.168454 e-03 \quad 7.215547 e-04$ $8.500000 e-033.946915 e-032.750413 e-03$ $9.000000 \mathrm{e}-03 \quad 1.105951 \mathrm{e}-02 \quad 8.494526 \mathrm{e}-03$ $9.500000 e-032.666542 e-022.130889 e-02$ $1.000000 e-024.625748 e-02 \quad 1.492872 e-02$ 45

5

$8.000000 e-03 \quad 6.210500 e-04 \quad 3.678873 e-04$ $8.500000 e-032.746342 e-031.915113 e-03$ $9.000000 \mathrm{e}-031.060803 \mathrm{e}-028.018221 \mathrm{e}-03$ $9.500000 e-033.327366 e-022.818675 e-02$ $1.000000 e-020.000000 e+007.692313 e-02$ 55

5

$8.000000 e-03 \quad 3.514479 e-04 \quad 1.940539 e-04$ $8.500000 \mathrm{e}-032.146034 \mathrm{e}-03 \quad 1.463901 \mathrm{e}-03$ $9.000000 \mathrm{e}-03 \quad 1.126806 \mathrm{e}-028.856805 \mathrm{e}-03$ $9.500000 \mathrm{e}-03 \quad 4.394984 \mathrm{e}-02 \quad 3.685991 \mathrm{e}-02$ $1.000000 e-021.363635 e-011.515150 e-02$
[1] P. W. Shor, in Proc. 35th Annual Symposium on Foundations of Computer Science (IEEE Computer Society Press, Los Alamitos, CA, 1994), pp. 124-134, quant$\mathrm{ph} / 9508027$.

[2] S. Lloyd, Science 273, 1073 (1996).

[3] S. Jordan, Quantum algorithm zoo, http://math.nist.gov/quantum/zoo/ (2012).

[4] D. S. Wang, A. G. Fowler, and L. C. L. Hollenberg, Phys. Rev. A 83, 020302(R) (2011), arXiv:1009.3686.

[5] A. G. Fowler, A. C. Whiteside, and L. C. L. Hollenberg, Phys. Rev. Lett. 108, 180501 (2012), arXiv:1110.5133.

[6] S. B. Bravyi and A. Y. Kitaev, quant-ph/9811052 (1998).

[7] E. Dennis, A. Kitaev, A. Landahl, and J. Preskill, J. Math. Phys. 43, 4452 (2002), quant-ph/0110143.
[8] R. Raussendorf and J. Harrington, Phys. Rev. Lett. 98, 190504 (2007), quant-ph/0610082.

[9] R. Raussendorf, J. Harrington, and K. Goyal, New J. Phys. 9, 199 (2007), quant-ph/0703143.

[10] A. G. Fowler, M. Mariantoni, J. M. Martinis, and A. N. Cleland, Phys. Rev. A 86, 032324 (2012), arXiv:1208.0928.

[11] A. G. Fowler, A. C. Whiteside, A. L. McInnes, and A. Rabbani, arXiv:1202.6111 (2012).

[12] J. Edmonds, Canad. J. Math. 17, 449 (1965).

[13] J. Edmonds, J. Res. Nat. Bur. Standards 69B, 125 (1965).

[14] D. Gottesman, Ph.D. thesis, Caltech (1997), quantph/9705052. 
[15] G. Duclos-Cianci and D. Poulin, Phys. Rev. Lett. 104, 050504 (2010), arXiv:0911.0581.

[16] J. R. Wootton and D. Loss, arXiv:1202.4316 (2012).
[17] S. Bravyi, G. Duclos-Cianci, D. Poulin, and M. Suchara, arXiv:1207.1443 (2012). 